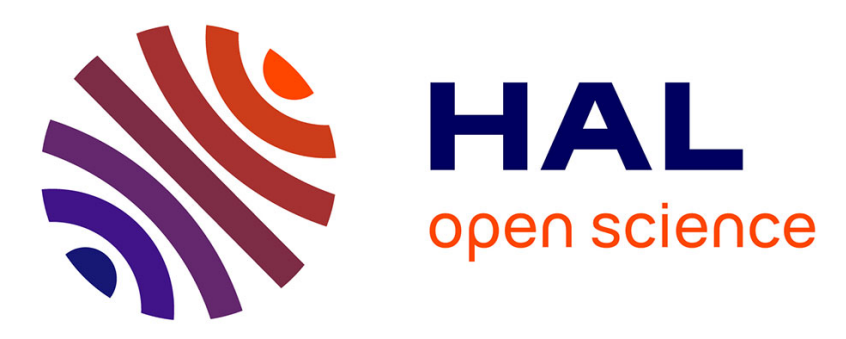

\title{
A Timed-Automata Based Middleware for Time-Critical Multicore Applications
}

Dario Socci, Peter Poplavko, Paraskevas Bourgos, Saddek Bensalem, Marius Bozga

\section{- To cite this version:}

Dario Socci, Peter Poplavko, Paraskevas Bourgos, Saddek Bensalem, Marius Bozga. A TimedAutomata Based Middleware for Time-Critical Multicore Applications. 11th International IEEE/IFIP Workshop on Software Technologies for Future Embedded and Ubiquitous Systems SEUS 2015, Jens Knoop, Apr 2015, Auckland, New Zealand. pp.1-8, 10.1109/ISORCW.2015.55 . hal-01212346

\author{
HAL Id: hal-01212346 \\ https://hal.science/hal-01212346
}

Submitted on 6 Oct 2015

HAL is a multi-disciplinary open access archive for the deposit and dissemination of scientific research documents, whether they are published or not. The documents may come from teaching and research institutions in France or abroad, or from public or private research centers.
L'archive ouverte pluridisciplinaire HAL, est destinée au dépôt et à la diffusion de documents scientifiques de niveau recherche, publiés ou non, émanant des établissements d'enseignement et de recherche français ou étrangers, des laboratoires publics ou privés. 


\title{
A Timed-automata based Middleware for Time-critical Multicore Applications
}

\author{
Dario Socci ${ }^{1}$, Peter Poplavko ${ }^{1}$, Paraskevas Bourgos, Saddek Bensalem ${ }^{1}$ and Marius Bozga ${ }^{2}$ \\ ${ }^{1}$ Univ. Grenoble Alpes, VERIMAG, F-38000 Grenoble, France \\ ${ }^{2}$ CNRS, VERIMAG, F-38000 Grenoble, France \\ $\{$ Dario.Socci $\mid$ Petro.Poplavko | Saddek.Bensalem | Marius.Bozga\}@imag.fr \\ Paraskevas.Bourgos@gmail.com
}

\begin{abstract}
The goal of our work is to contribute to unification of design methodologies for multi-core time-critical systems. Various models of computation have been proposed in literature for this kind of systems, but lack of coherency between them makes unified coherent design methodology challenging. In addition, there is a significant gap between the models of computation and the real-time scheduling and analysis techniques. To overcome this difficulty, we represent both the models of computation and the scheduling policies by timed automata. While, traditionally, they are only used for simulation and validation, we use the automata for programming. We believe that using the same formal language for different design styles and methods is an important step to close the gap between them. Our approach is demonstrated using a publicly available toolset, an industrial application use case and a multi-core platform.
\end{abstract}

\section{INTRODUCTION}

The formal techniques help to address different challenges in real-time system design. One of them is lack of consolidation in programming. Embedded software design has in common with hardware design that it has to satisfy not only functional, but also extra-functional requirements, first of all, timing. However, unlike hardware languages, the software languages have an important deficiency: they were conceived without any concern on timing in mind [1]. The real-time programming is a very heterogeneous area of research, as it employs many different models of computation (MoCs), such as synchronous languages, timed Petri nets, various extensions of synchronous dataflow (SDF), etc. Expressing the software design in a given $\mathrm{MoC}$ is difficult, but, worse still, even when this is done, the real-time scheduling and timing analysis still remains challenging, due to a gap between the MoCs and the real-time scheduling policies [2].

Therefore it takes a lot of effort to compose a middleware by combining a particular model and particular policy. This task could be simplified if there existed a common 'backbone' language expressive enough to redefine and reuse different components of middleware. Partly, this idea was implemented in the SystemC project, offering a common way to express scheduling policies, MoCs and functional code. However, this language lacks a formal semantics, and it mainly offers facilities for simulation only, but not for fully-automated deployment of software.

Therefore, as an alternative, we propose to use combined procedural and automata languages. To demonstrate

Research supported by the European ICT Collaborative Project no. 288175 (CERTAINTY) and no. 332987 (ARROWHEAD). the concept, we offer public prototype tools [3] for multicore timing-critical system design based on the timed-automata language RT-BIP [4]. This paper describes the design flow for programming and implementing timing-critical systems in this language. Section II gives an overview of the proposed design flow, Section III gives an introduction into RT-BIP, then in Section IV we show how to translate an high-level description of the software, including the middleware, into RTBIP. In Section V we describe implementation of an industrial use case on Kalray MPPA256 multi-core platform and some experimental results. Finally, Section VI concludes the paper.

\section{BACKGROUND}

In this section we present our proposed design flow. It is based on a combined procedural and automata-based language, referred to as backbone language. Examples of such languages are IF, SDL-RT and RT-BIP. A backbone language can be used for modeling, validation and simulation, but our main point is to use it as a programming language. Because in many cases, an automata-based language may be too low-level for direct use in application programming, we can compile higher-level models into the backbone language automatically. In the ideal case, this is ensured by letting the user create a set of rules for automatic translation of the functional code into the automata. Also the user would provide a set of automata templates that implement the primitives of the preferred MoC and scheduling policy. Hence the backbone language would serve as a metamodel and meta-policy used to program the desired timingcritical systems middleware. The specified set of rules and templates would allow to compile the functional code and the middleware into a network of timed automata that can be analyzed and deployed on a platform.

This idea is partly implemented in our design flow, see Fig. 1. The design flow accepts a high-level specification of application tasks (the $\mathrm{MoC}$ and the functional code) at the input and compiles it into the backbone language, for which we use RT-BIP [4]. Also the flow takes from the offline scheduling tool the specification of the online scheduling policy and the selected scheduling parameters (such as priorities) of the tasks. The scheduler is also compiled into backbone language and 'plugged' into the common RT-BIP software model. This model is deployed on the platform on top of RT-BIP runtime environment (RTE) for multi-cores. The software model can also be combined with the hardware model to represent the complete software-hardware system and to perform timing analysis for validation of schedulability properties, but the 


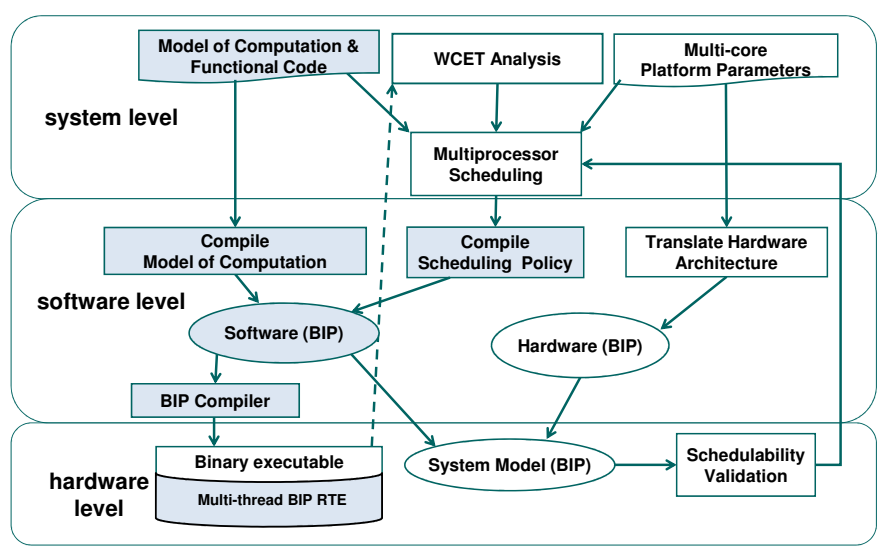

Fig. 1. Design flow (highlighting the steps covered in this paper)

validation part of the design flow is beyond the scope of the prototype toolset [3] and this paper.

Currently we support only one MoC - Fixed Priority Process Networks (FPPN) [5], which combines the abilities to model both the reactive-control and streaming applications. As for the scheduling policies, we support a combined staticorder/time-triggered policy [6], [5]. In future we consider to provide means to the user to specify templates for his preferred $\mathrm{MoC}$ and policy. We also consider to add support for other relevant MoCs, such as synchronous languages, as in the Prelude [7] framework, and SDF, such as in CompSoC [8].

\section{Backbone Language: Real Time BIP}

Our backbone language is RT-BIP [4]. The "RT" prefix stands for real-time, which indicates that this language models the physical time, which is done using the same concept of clocks as in timed automata. In fact, the RT-BIP language can be seen as a language to express networks of communicating timed automata that employs a specific compositional syntax and a specific flavor of timing constraints. The "BIP" part of the acronym stands for behaviour-interactions-priority.

In this section, we give an overview of the particular dialect of RT-BIP that we use in our framework for timing-critical multi-core applications. Mainly, this dialect is a restriction of RT-BIP. We also add to the standard RT-BIP a new concept - the continuous transitions, which, unlike the standard RTBIP transitions, are non-instantaneous. Fig. 2 shows an RTBIP example that represents two tasks, $\mathrm{A}$ and $\mathrm{B}$, running on two CPUs. The model consists of four components, namely, "PeriodicA", "DelayableB", "CPU1" and "CPU2". All the components are defined by an automaton and a set of ports.

The states of the BIP components are usually referred to as locations. A transition is an execution step from one location to itself or to another location. For example "(Skip)" is a transition from "S1" to "S0" in component "DelayableB".

Each transition has an associated enabling condition and an associated action that is executed at this transition. In our figures we show the conditions in blue color and in square brackets, e.g. condition $\left[D_{O U T} \neq 0\right]$ for transition "StartB" in "DelayableB". In BIP, every component is seen as an object in object-oriented programming sense. Every component encapsulates some data and some methods to manipulate the data. All actions executed by transitions can execute methods

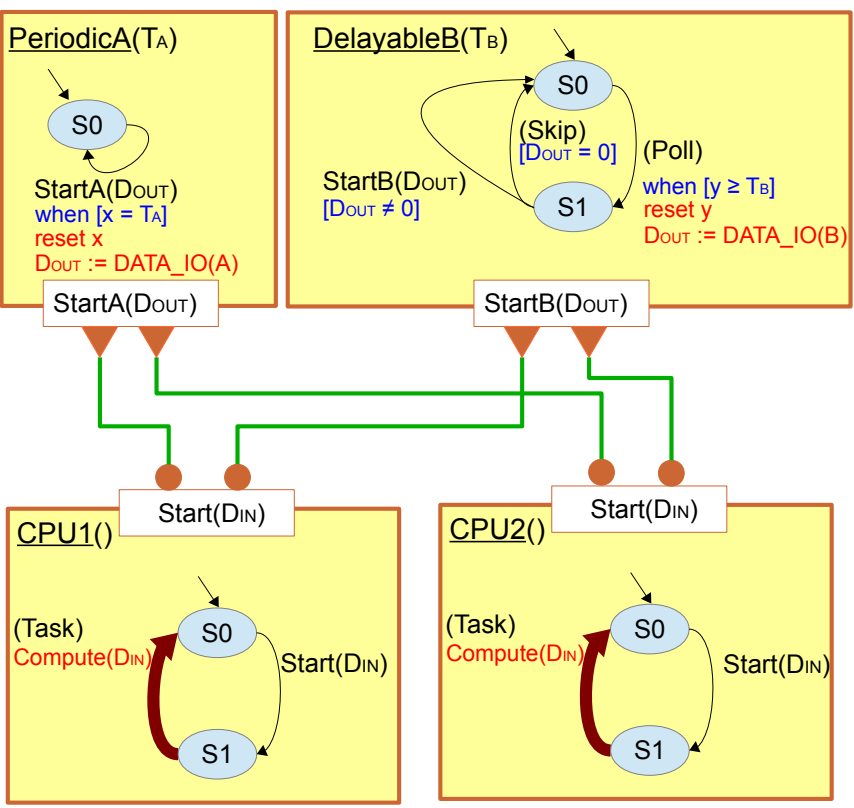

Fig. 2. RT-BIP model example

written in an imperative language $(\mathrm{C} / \mathrm{C}++)$. The methods have access only to the local variables of the component itself, the components do not share variables. In the figures the actions are shown as blocks of pseudo-code in dark-red color.

Multiple components run concurrently and execute interactions with each other, the set of possible interactions being defined using connectors (shown in green lines) which join several ports (enclosed in white rectangles in our figures), which belong to components. In our RT-BIP dialect we use only one type of BIP connector, "rendezvous", which obliges all connected ports to participate in an interaction simultaneously. A port participating in interaction means that a transition annotated by the given port gets executed. This can only happen when the transition is enabled, i.e., the automaton current state is the source location of the transition and all conditions associated to the transition are satisfied. For example, port "StartB" can participate in interaction only when the automaton in the "S1" location and "DOUT $\neq 0$ ". A port may participate in one interaction at a time. If in our example all four ports are enabled then four interactions can potentially occur, but only two of them will be selected (in non-deterministic way).

A transition can be either internal or external. Internal transitions are not annotated by ports and do not participate in interactions with other components. We enclose their annotations in parentheses to distinguish them, e.g. "(Task)" in our example. External transitions are annotated with a port, such as transition "StartB" in "DelayableB". The same port can be annotated on multiple transitions. An external transition executes simultaneously with an interaction at the corresponding port. Note that in our figures we sometimes annotate a port by a circle (e.g., "Start $\left(D_{I N}\right)$ ") or a triangle (e.g., "StartB $\left(D_{O U T}\right.$ ")). These annotations do not refer to any construct in the BIP language but indicate one of the two roles that the port typically plays in the interactions by construction of the model. The triangle means a "master" port and the circle means a "slave" port. A master joined by a connector to several 
slaves indicates that the slaves are supposed to be enabled whenever the master may get enabled. This means that it is the master who "takes the initiative" for the interaction and at the moment when it is ready to execute the whole interaction can execute immediately. The master and slave notations in our figures serve only to improve the readability of the figures.

Each component has an initial transition that is executed at start of execution, shown as an arrow without a source location pointing to one of the locations, which makes it the initial location, such as location "S0" in "DelayableB". Each component has an associated set of private local variables: data variables and clocks. In line with the usual conventions adopted in timed automata, the clocks are real-valued variables that are initialized to zero at start and whose values are continuously increasing with the passage of physical time. In our models we use letters $x, y$ for the clocks, e.g. the model in Fig. 2 uses two clocks. A clock can be reset to zero at a transition (e.g. "reset $x$ " in "PeriodicA") and used in a condition, in which case the condition is preceded by the word "when".

We use only urgent timing conditions (eager constraints in RT-BIP). All transitions are eager in our model, which means that they execute at the earliest moment of time when they are enabled for execution. For an external transition this means the earliest moment of time when all ports participating in an interaction with its port are enabled. For example, consider condition "when $\left[y \geq T_{B}\right]$ " in Fig. 2. Due to this condition the interactions involving port "StartB" should wait the earliest time when clock $y$ reaches value at least $T_{B}$.

Though the transitions start urgently, another issue is when they finish. The instantaneous transitions take zero time (conceptually). By default, all RT-BIP transitions are instantaneous, with exception of those that have continuous attribute. The latter transitions, the so-called continuous transitions take exactly the time required to execute the corresponding action, which can be any timing duration not known at the moment when the transition starts. Only internal transitions can be given a continuous attribute. In our figures we denote continuous transitions by thick arrows, e.g., "(Task)" transitions in our model. In modeling the real-time tasks, we use such transitions to represent the blocks of execution where the does not interact with the runtime environment but is performing internal computations instead, e.g., running the "Compute()" method in our example in Fig. 2.

As for the data variables, in our examples we most often use three types: integer (such as $D_{I N}, D_{O U T}$ in our example), Boolean and queue, though, of course, other types can be defined in RT-BIP using C/C++ syntax. Unless explicitly done otherwise in the action of the initial transition, we assume that the initial transition implicitly initializes the data variables to zero in the case of integers, "False" in the case of Booleans, and type-specific initial value (such as empty queue) for other types. Next to data variables there are also compile-time parameters, for example, period $T_{A}$ and minimal execution interval $T_{B}$ in Fig. 2.

Some variables are used for communication between the components at the interactions. They are sent and received via ports, therefore they are listed as port parameters. We assume that if a port has parameter $<$ name $>_{O U T}$ then an interaction of this port assigns the value of this variable to the variables
$<$ name $>_{I N}$ with the same $<$ name $>$ if they are parameters of other ports that participate in the interaction. For example, port $\operatorname{Start}\left(D_{I N}\right)$ receives the value of $D_{I N}$ from the $D_{O U T}$ of either "PeriodicA" or "DelayableB".

In our RT-BIP programs for time-critical systems we often use queues. This well-known data structure can be easily implemented using a circular buffer. We define the following operations on the queue:

- Allocate() allocate a new cell and give reference to it

- Push() push the last allocated cell into the tail

- $\boldsymbol{P o p}()$ extract the head of the queue

\section{COMPILING ThE SOFTWARE INTO RT-BIP}

The time-critical software consists of functional code and middleware, the latter providing elements for communication, synchronization and real-time scheduling. Compiling means translating the functional code and middleware specification into components of RT-BIP language and connecting them with each other. The components express the correct timing behavior by timing constraints and transitions. A situation where for a component automaton no transitions are possible anymore in future is called local deadlock and is detected as a runtime error. The RT-BIP components generated at compilation are constructed in such a way that a deadlock indicates that that either the hardware resources cannot handle the workload on time or that the workload does conform to specification. For example, in Fig. 2, component "PeriodicA" is ready to execute an interaction at port "StartA" only when $x=T_{A}$. If at this moment of time both "CPU"components are busy executing the previously started "(Task)"'transitions, then component "PeriodicA" will deadlock as the clock $x$ will continue counting the time, never come back to $T_{A}$. To avoid a deadlock in "PeriodicA", at least one of the "CPU" components should be ready for interaction at periodic instances in time: $T_{A}, 2 T_{A}, 3 T_{A}, \ldots$. Similar conditions hold for certain BIP components generated at compilation.

\section{A. The FPPN Model of Computation}

In our framework, we currently work with a functionallydeterministic MoC intended to support both the reactive control and the streaming applications, the so-called Fixed Priority Process Networks (FPPN) [5]. An instance of FPPN is composed of three main entities: Processes, Data Channels and Event Generators. The determinism is ensured by Functional Priority relation between the processes.

A Process represents a software subroutine that operates with internal variables and input/output channels connected to it through ports. The functional code of the application is defined in processes, whereas the necessary middleware elements of FPPN are channels, event generators, and priorities.

An example of process is given in Fig. 3. This process example performs a check on the internal variables, then (if the check is successful) reads from the input channel, and, if the value is valid (see channel description below) makes the square of it. Then the write operation on an output channel is performed. A single invocation of the subroutine that defines the process is referred to as as a job. Like the real-time jobs, this subroutine should have a bounded execution time and is subject to WCET (worst-case execution time) analysis. 


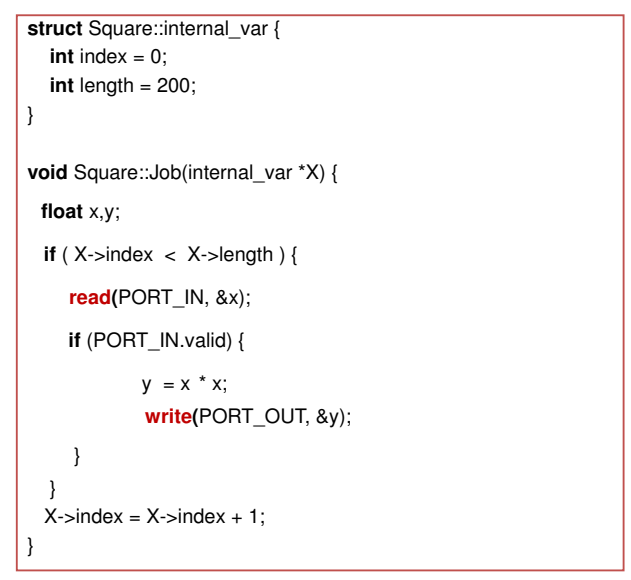

Fig. 3. Functional Code for "Square" Process Example

Data Channels ensure non-blocking read and write operations for communication. There are inter-process and external (environment) channels. In this paper we consider only the inter-process channels. We define two channel types: FIFO and blackboard. Other types can be introduced by extension of the library of RT-BIP components. The FIFO has a semantics of a queue. The blackboard remembers the last written value that can be read multiple times. Reading from an empty FIFO or a non-initialized blackboard resets an indicator of data validity.

An event generator $e$ is defined by the set of possible sequences of time stamps $\tau_{k}$ that it can produce. We define two types of event generators: periodic and sporadic. Every event generator is associated with a unique process and determines whether the given process is periodic or sporadic one. Every process $p$ has a deadline $d_{p}$. Interval $\left[\tau_{k}, \tau_{k}+d_{p}\right)$ determines the time interval when the $k$-th process job can be executed. At $\tau_{k}$ the job gets 'activated' and then it remains active until it is scheduled. After being schedule, the job should terminate before the deadline. Periodic processes are activated at period $T_{p}$, for sporadic processes $T_{p}$ denotes the minimal inter-arrival time. We define the job queue length as $q_{p}=\left\lceil d_{p} / T_{p}\right\rceil$, this quantity is the maximum number of jobs of process $p$ that can be active simultaneously.

An FPPN network can be described by two directed graphs. The first graph is the default process network graph $(P, C)$, whose nodes are processes $P$ and the edges are channels $C$. This graph can be cyclic and defines the communicating pairs of processes and the direction of dataflow: from writer to reader. The second graph is the functional priority DAG: $(P, F P)$. No cyclic paths are allowed in this graph. The edges define functional priority relation between the processes. It is however, not a partial order relation, as it is not necessarily transitive. We require that any two communicating processes have a priority relation: if $\left(p_{1}, p_{2}\right) \in C$ then $\left(p_{1}, p_{2}\right) \in F P$ or $\left(p_{2}, p_{1}\right) \in F P$, i.e., a functional priority should either follow the direction of the data flow or the opposite direction.

Fig. 4 below gives an example of a process network. This process network represents an imaginary signal processing application with input sample period $200 \mathrm{~ms}$, reconfigurable filter coefficients and a feedback loop. The filter coefficients are reconfigured by an sporadic event (a command from the environment) that activates the sporadic process CoefB.

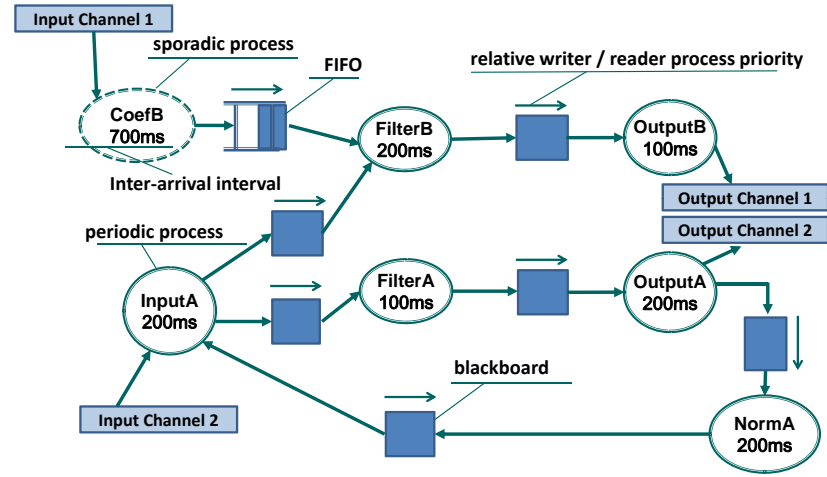

Fig. 4. Example of Process Network

We see several periodic processes, annotated by their periods, and a sporadic process, annotated by minimal inter-arrival time. We also see inter-process channels - the blackboards and a FIFO, annotated by an arc of the functional priority relation FP. Also the environment input/output channels are shown.

The semantics FPPNs is described in [5]. The main idea is that every pair of processes that share a channel are executed in well-defined relative order determined by (1) their activation times and (2) functional priorities. This order is compatible to the total order derived from zero-execution-time simulation of fixed-priority scheduling. Because the ordering is imposed only between communicating processes, it is a partial order, allowing for parallelism.

\section{B. Compiling the processes}

The BIP model of a process is automatically extracted from its source code. When translating from a FPPN to a BIP model, the source code is parsed, searching for primitives that are relevant for the interactions between the process and the other components of the system. The relevant primitives are the reads and writes from/to the data channels. Fig. 5 shows the result of compiling the process of Fig. 3 into the backbone language. It can be seen that the behaviour of the resulting automaton is consistent with the behavior of the original source code. The most important difference is how the "reads" and "writes" are performed. As shown in the figure, each I/O operation is divided into three transitions. Let us consider the read transitions for example. First we have a "Read_Req", which is an external transition which requests access to the channel. After the corresponding interaction the process receives a reference to a memory area where to read from and a validity flag. The next transaction performs the actual read if the validity flag is true. Then transaction "Read_Ack" communicates to the channel that the read has finished. The writing is performed in a similar way.

\section{Compiling the Scheduling Policy}

Our RT-BIP run-time environment (RTE) currently does not support the interruption of running transitions. Therefore, in our current middleware for time-critical systems we do not yet support preemption. It should be noted that many multi-core platforms choose to not support preemption, instead providing a large number of cores to ensure sufficient degree of multithreading concurrency without preemption. 


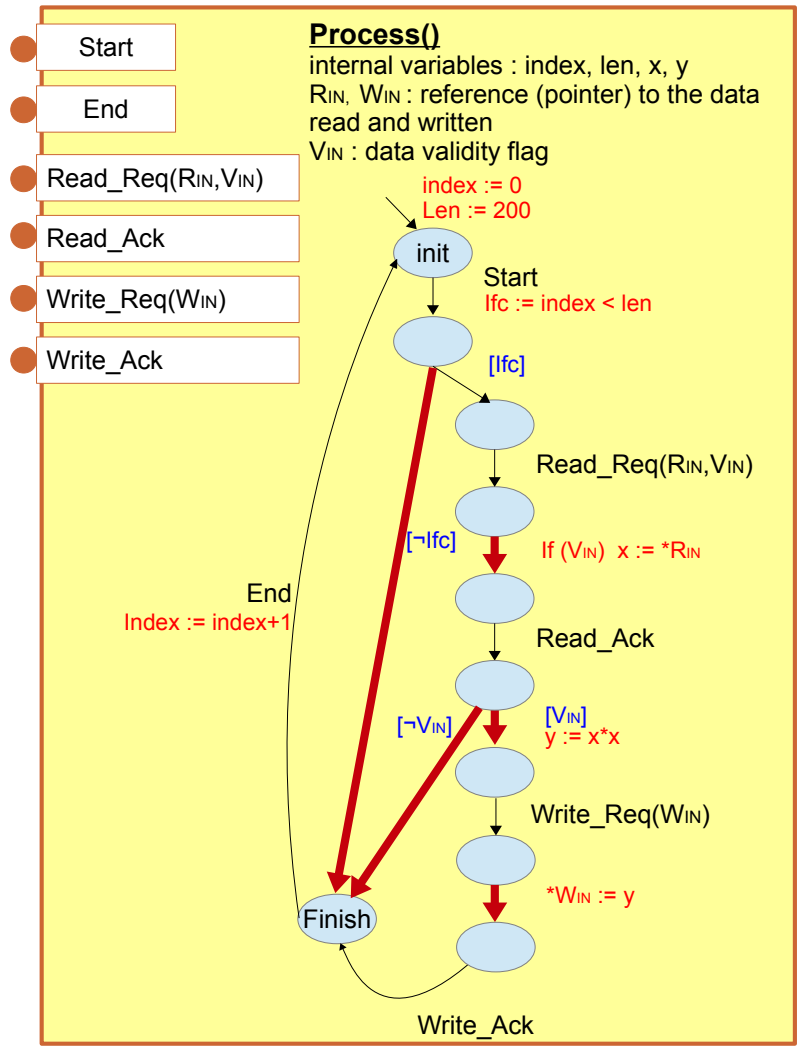

Fig. 5. Process translated in BIP
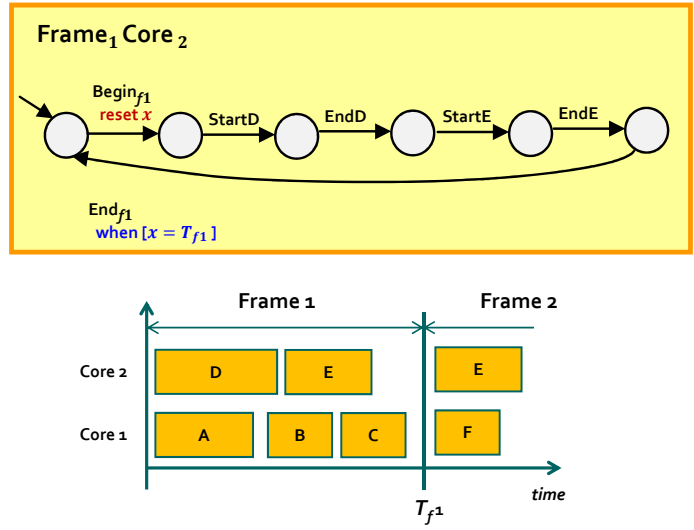

Fig. 6. Scheduling Frames

We demonstrate programming the scheduling policies in timed automata by considering a policy that combines staticorder execution and time-triggering. We base our policy on the policies presented in [6] and partly in [5]. We divide the execution of the FPPN in several slices called "Frames". To each core and each time frame the offline scheduling tool associates a list of jobs that must execute sequentially on the given core in the given time frame. The online schedule is a periodically repeating cycle, where all frames are executed in order: $f_{1}, f_{2}, \ldots f_{I}$, on all cores in parallel, every frame having a fixed duration $T_{f 1}, T_{f 2}, \ldots$..

In the Gantt chart of Fig. 6 we can see a partial example

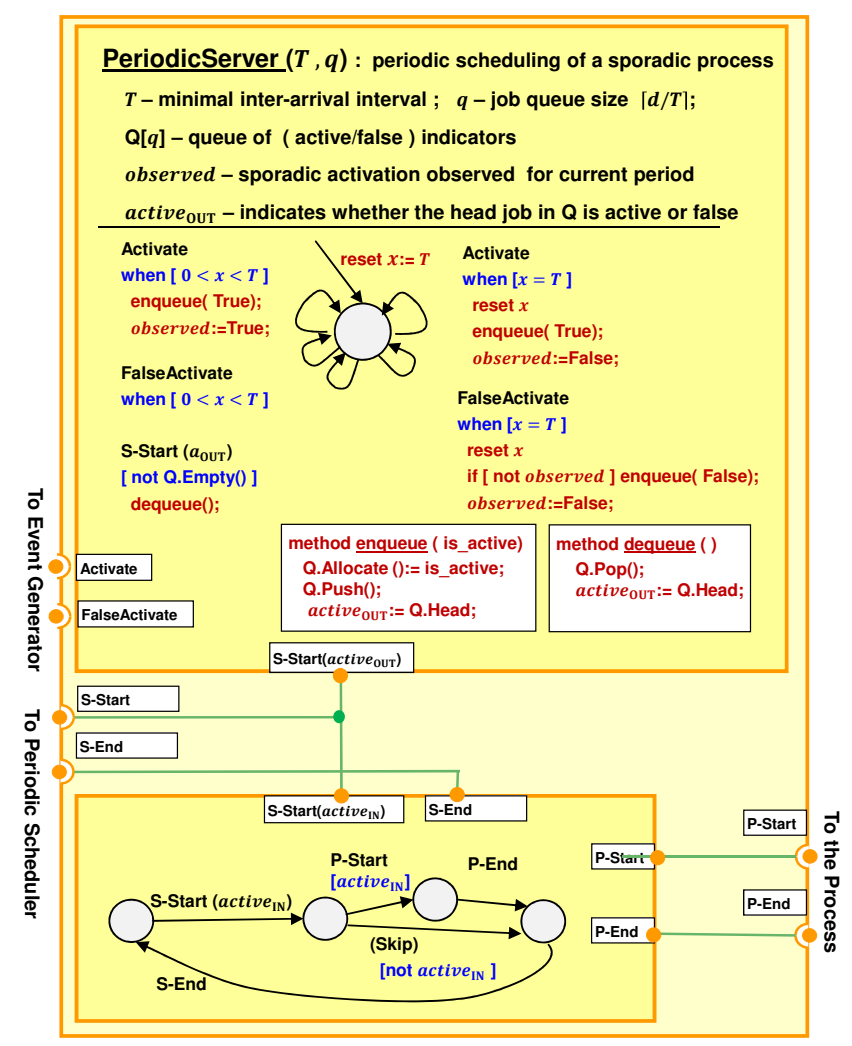

Fig. 7. Periodic server, consisting of two sub-components

of a schedule. The BIP implementation of the "Frame 1" of "Core 2" is also shown. The frame components in BIP are specific per frame $i$ and core $k$. In the beginning, the component synchronizes with other frame components for the same frame $i$ and other cores by interaction $\operatorname{Begin}_{f i}$. The jobs are scheduled in a given static order and they all should terminate before $E n d_{f i}$ interaction, which should occur exactly at time $T_{f i}$, otherwise the component will deadlock and a run-time error will occur.

In Fig. 7 the periodic server is shown, which is a supplementary adaptor for sporadic processes scheduled in periodic frames. This component manages a queue of active jobs. When a job is activated, it is inserted in the queue, and it is removed when it is scheduled. The queue may contain "false" jobs. This is used for scheduling purposes. We explained above that in each frame we execute jobs in a sequential way. If a frame contains a sporadic job, and this job does not activate, we could have a deadlock in the frame. Thus, to avoid this problem, whenever a sporadic process is not activated, we introduce in the queue a "false job" with zero execution time. The bottom sub-component of the periodic server in Fig. 7 distinguishes between "active" and "false" jobs. In the case of an active job, it signals the job start to the scheduler frame, then to the process, then it waits for the job termination and finally signals it to the frame. In case of a false job, the execution of the process job is skipped. A more detailed explanation of handling sporadic jobs by periodic server can be found in [5].

Fig. 8 shows how scheduler frames and a sporadic process are connected. The Event Generator generates the activation 


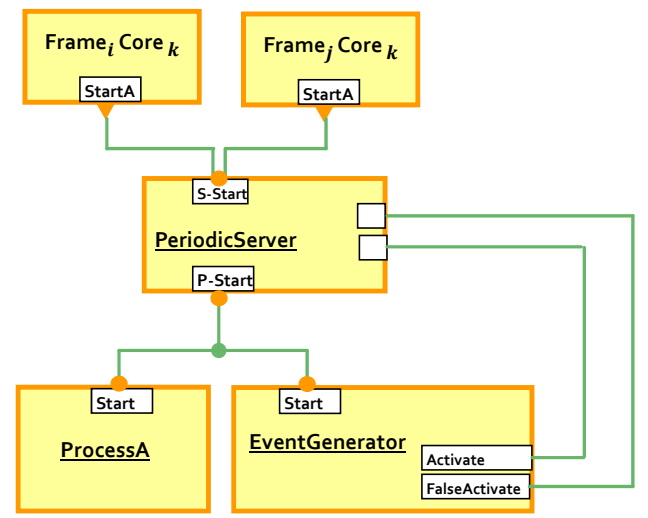

Fig. 8. Connection between a Sporadic Process and its Scheduler

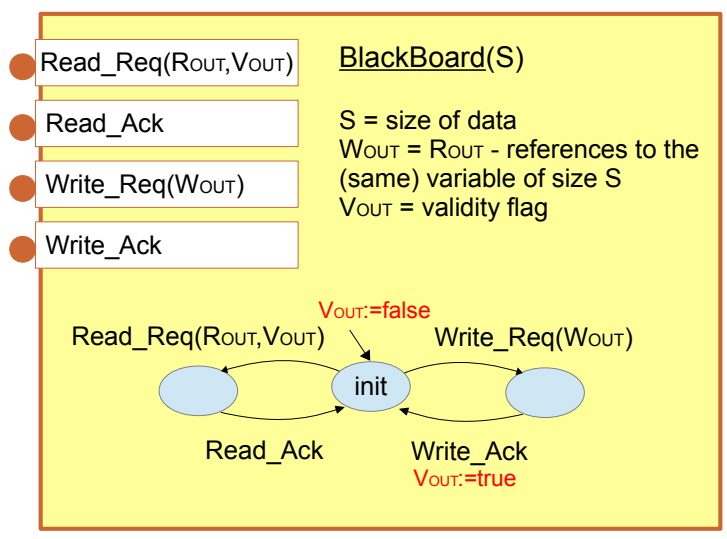

Fig. 9. Blackboard

signal and sends it to the Periodic Server, which triggers the Process in the order defined by the frames. For a periodic process, the periodic server is not necessary and the process can be connected directly to its scheduler and generator.

\section{Compiling the Inter-process Channels}

We present here the BIP components that model the data channels, used to "read" and "write" the data communicated between the processes. A basic notion in data channels is the validity bit, introduced earlier. The meaning of this bit is availability of data. In the two types of data channels, the blackboard and the FIFO, it is managed in a slightly different way. The blackboard represents a shared variable, for which it holds by default that once data is written there, it remains available (and hence valid) until it is overwritten by new data.

In the FIFO data items are read and removed in the same order as they are written, and an attempt to read from an empty "queue" or to read more data items than currently waiting in the queue leads to a result whose validity bit is set to "false".

Fig. 9 shows the model for the blackboard. Read(Write) operation is separated into two transactions Read_Req (Write_Req) and Read_Ack(Write_Ack), coherently to the process model shown above. During the request the blackboard communicates to the process the address of the memory where

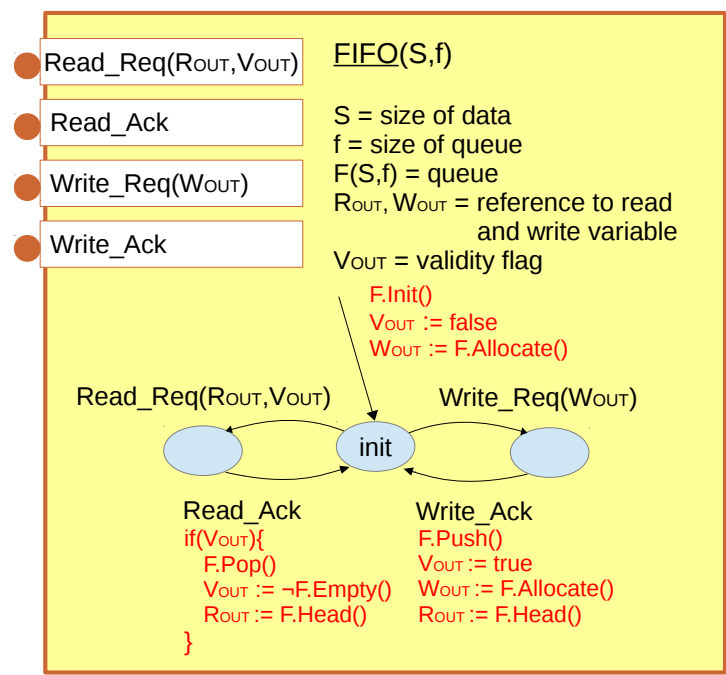

Fig. 10. FIFO

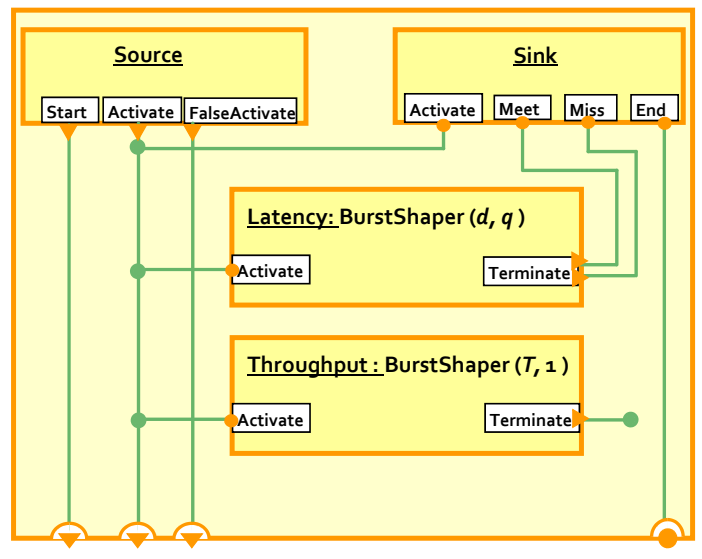

Fig. 11. Event Generator

to read from/write to. In case of a read, the validity bit is communicated as well.

The BIP model of a FIFO is shown in Fig. 10. We use the queue data structure introduced in Section III. The FIFO model is very similar to the blackboard, the main difference is in that here the code for managing the queue is added.

\section{E. Compiling the Event Generators}

We describe here the Event Generator component, individual for each process. The main purpose of this component is to enable the start of jobs after their activation. It also manages the "false" activation for sporadic jobs. The idea of the latter is that for sporadic process $p$ at small intervals $\delta=T_{p} / K$ for some integer $K$ the environment is polled for the need to activate the sporadic process by calling some platform-dependent subroutine function $\operatorname{protocol}()$ that returns a Boolean value indicating activation ('true') or false activation ('false'). The point is that ensure functional determinism in FPPN MoC, at each moment of time when a sporadic process may potentially get activated it should be always explicitly signaled whether it is activated or not. For periodic processes $\delta=T$ and $\operatorname{protocol}()$ always returns 'true'. 


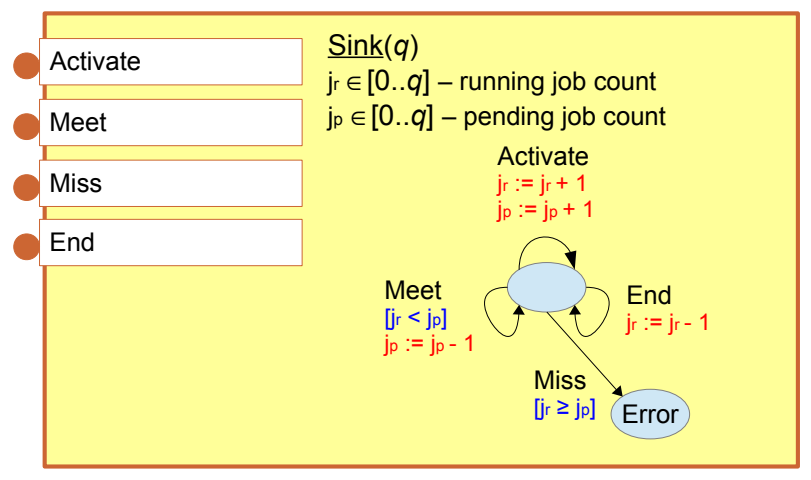

Fig. 12. Sink

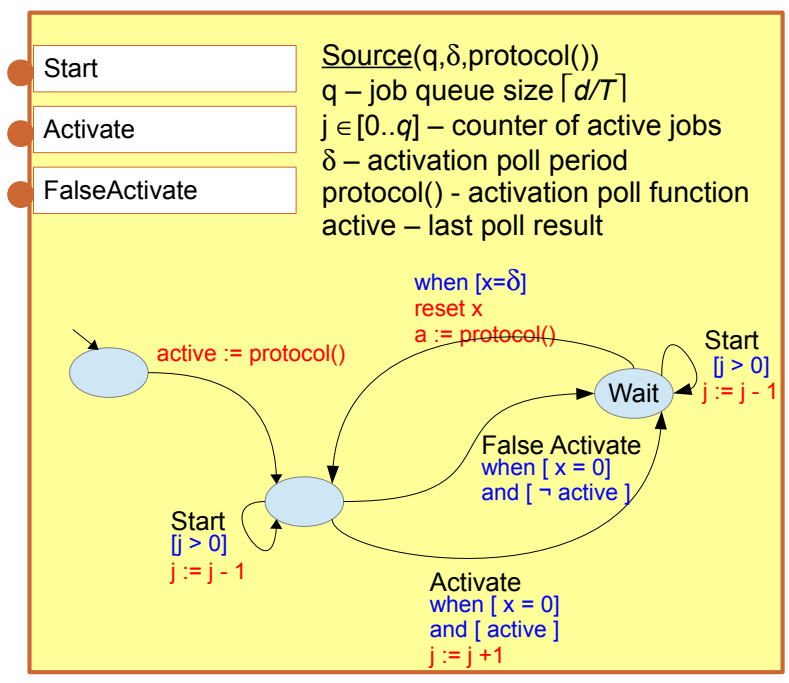

Fig. 13. Source

The event generator is shown in Fig. 11. It contains a few subcomponents. The Source triggers periodically or sporadically (depending on its protocol) the activation signal. The Sink checks whether any job misses its deadline. For this it uses "Latency - Burst Shaper" component, which can be seen as a delay line of delay $d_{p}$ and capacity up to $q_{p}$ events, where deadline and queue size are process parameters. At activation, the burst shaper starts a new timer (a clock), and when the deadline time was elapsed it enables the output. As shown in Fig. 12, the sink checks whether before the end of the deadline interval at least one job has terminated (pending and not running) or not and goes into local deadlock state if not (and this leads to runtime error).

The "Throughput - Burst Shaper" - ensures that the source cannot activate the jobs more than once per time $T_{p}$. This subcomponent can be omitted for periodic processes.

The implementation of Source is shown in Fig. 13. This component polls the "protocol()" at periodic intervals $\delta$, as explained earlier. After activation it enables another job to be started by incrementing the job counter.

Fig. 14 shows how a Burst Shaper is implemented. Its main purpose is to limit the amount of burst to at most $\sigma$ events per

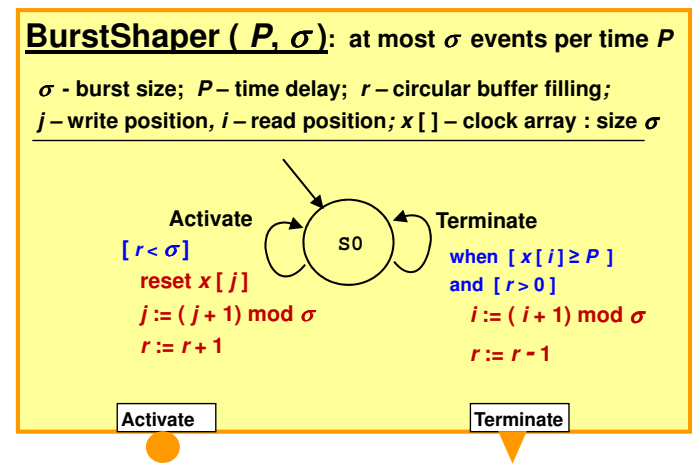

Fig. 14. Burst Shaper
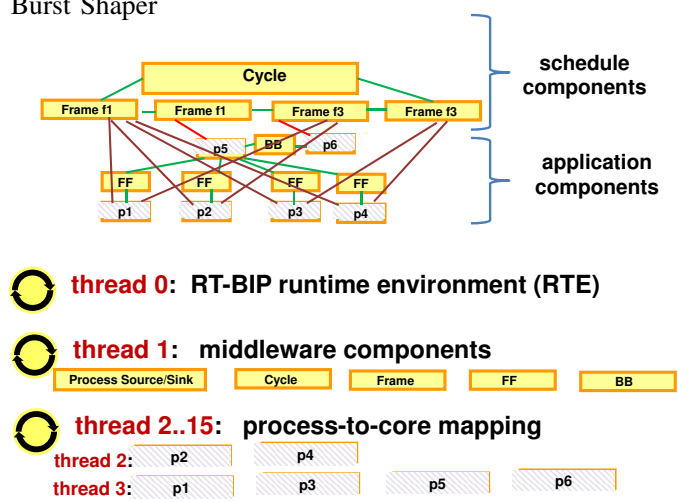

Fig. 15. Distributing BIP Components between Cores

time $P$ where $\sigma$ and $P$ are given during the definition of the component. This component also signals when an event that arrived $P$ time units ago has elapsed (terminated). The main idea of implementation is to use a queue of clock variables implemented as circular buffer.

\section{IMPLEMENTATION AND EXPERIMENTS}

\section{A. Multi-threaded RT-BIP Runtime Environment}

As illustrated in Fig. 15, after compiling the application and scheduling into BIP, the BIP design can be partitioned into parts: the schedule components and the application components. The components are joined by BIP connectors, through which they can perform interactions with each other. The application components include the components dedicated to FPPN processes, denoted $\mathrm{p} 1, \mathrm{p} 2, \ldots$, and data channels, denoted BB, FF, depending on the type: blackboard and FIFO. The schedule components include one component that models the schedule cycle and a set of components that model frames. The schedule components are connected to the application components to coordinate their execution according to the schedule. The schedule also provides the process-to-core mapping, which is used to generate component-to-thread mapping, illustrated in the bottom part of the figure.

We implemented our framework on Kalray MPPA multicore architecture inside a single shared-memory cluster. The cluster provides 16 processor cores, each one running one POSIX thread. In our framework, Thread 0 executes the RTBIP run-time environment (RTE), which coordinates the components for the execution according to the RT-BIP semantics. Then, on Thread 1 we run all the middleware components, 

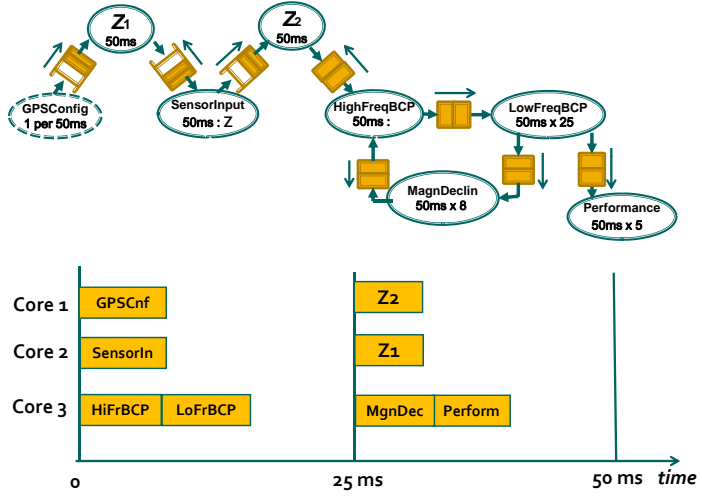

Fig. 16. FMS Use Case and its Static Schedule

i.e. all components except the processes. Note that those components can be seen as "instantaneous" components, as they execute only instantaneous transitions. We thus isolate them in a separate thread from the processes, to avoid the risk that continuous transitions of the processes delay the instantaneous transitions of the middleware. Finally, Threads 2..15 are reserved to the process components, which are distributed according to the static schedule computed by an offline scheduling tool.

\section{B. Experiments}

In this experiment we consider a subsystem of avionics Flight Management System (FMS). Figure 16 shows the application process network. This FMS subsystem is responsible for calculating the best computed position (BCP) and predicting the performance (e.g., fuel usage) of the airplane based on the sensor data and sporadic configuration commands from the pilot, such as configuring the Global Positioning System (GPS). Therefore we have a sporadic process GPSConfig that can execute at most once per $50 \mathrm{~ms}$.

After being pre-processed at "SensorInput", the input data is processed at "HighFrewBCP" process and arrives at "LowFreqBCP" process, which post-processes the data at low frequency and makes it available by other subsystems of FMS. It also provides the results to a feedback loop that takes into account magnetic declination in computing the airplane position. All periodic processes are triggered by event generators and scheduler at period $50 \mathrm{~ms}$, but some of them internally skip every $k$-th execution to execute their true frequency in this multi-rate design. This is not because we do not support different process periods, but to simplify the static schedule.

To enable pipelining parallelism we use a double buffer approach and insert at some places of the process network processes denoted as $Z_{k}$ which copy input to the output. Because of this, the buffer is split into two parts and thus the writer can execute at the same time as the reader.

The measured Gantt chart of the execution traces is shown in Figure 17. Studying the chart we conclude that we succeeded in correctly implementing the parallel schedule, but the BIP RTE component synchronization actions turn out to have a large overhead due to inter-core synchronization and cache

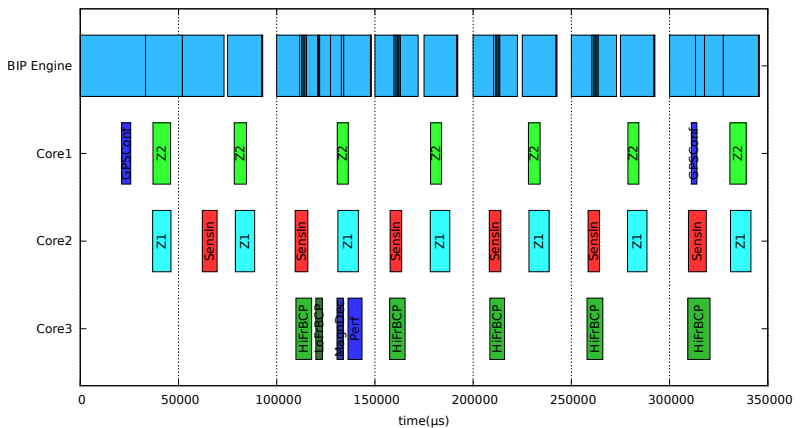

Fig. 17. FMS Use Case

flushing for memory consistency that occur at BIP interactions. In future work, we see a large room for improvement to reduce this overhead. Note that this overhead is relatively large compared to process execution because of fine granularity of the process computations, for more coarse-grained processes the relative overhead of BIP interactions would be smaller.

\section{CONCLUSIONS}

In this paper we proposed a common approach to program not only application functionality, but also the middleware for time-critical systems. We proposed to use for this purpose combined timed-automata/procedural languages that support deployment on multi-cores in multiple threads. We demonstrated this approach on a concrete model of computation and scheduling policy, and implemented it in a publically available tool [3]. The proposed approach potentially opens the possibility of unifying different MoCs and scheduling policies in common frameworks. To confirm the validity of our approach we showed the results for deploying a real-life avionic use-case on a real multi-core platform.

\section{REFERENCES}

[1] E. A. Lee, "Absolutely positively on time: what would it take?[embedded computing systems]," Computer, vol. 38, no. 7, pp. 85-87, 2005.

[2] H. Fuhrmann, J. Koch, J. Rennhack, and R. von Hanxleden, "Modelbased system design of time-triggered architectures-an avionics case study," in 25th Digital Avionics Systems Conference (DASC'06), (Portland, OR, USA), October 2006.

[3] P. Poplavko, P. Bourgos, D. Socci, S. Bensalem, and M. Bozga, "Multicore code generation for time-critical applications, http://wwwverimag.imag.fr/multicore-time-critical-code,470.html."

[4] T. Abdellatif, J. Combaz, and J. Sifakis, "Model-based implementation of real-time applications," in Proceedings of the tenth ACM international conference on Embedded software, EMSOFT '10, ACM, 2010.

[5] P. Poplavko, D. Socci, S. Paraskevas Bourgos, and M. B. Bensalem, "Models for deterministic execution of real-time multiprocessor applications," in DATE'15, 2015.

[6] G. Giannopoulou, N. Stoimenov, P. Huang, and L. Thiele, "Scheduling of mixed-criticality applications on resource-sharing multicore systems," in Embedded Software (EMSOFT), 2013 Proceedings of the International Conference on, pp. 1-15, IEEE, 2013.

[7] M. Cordovilla, F. Boniol, J. Forget, E. Noulard, and C. Pagetti, "Developing critical embedded systems on multicore architectures: the preludeschedmcore toolset," in 19th International Conference on Real-Time and Network Systems, 2011.

[8] A. Hansson, K. Goossens, M. Bekooij, and J. Huisken, "Compsoc: A template for composable and predictable multi-processor system on chips," ACM Transactions on Design Automation of Electronic Systems (TODAES), vol. 14, no. 1, p. 2, 2009. 